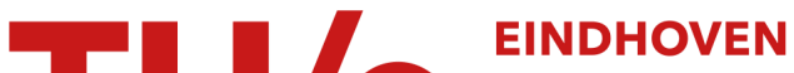 \\ UNIVERSITY OF \\ TECHNOLOGY
}

\section{Some theorems on Gabor operators}

\section{Citation for published version (APA):}

Graaf, de, J. (1981). Some theorems on Gabor operators. (Eindhoven University of Technology : Dept of Mathematics : memorandum; Vol. 8102). Technische Hogeschool Eindhoven.

\section{Document status and date:}

Published: 01/01/1981

\section{Document Version:}

Publisher's PDF, also known as Version of Record (includes final page, issue and volume numbers)

\section{Please check the document version of this publication:}

- A submitted manuscript is the version of the article upon submission and before peer-review. There can be important differences between the submitted version and the official published version of record. People interested in the research are advised to contact the author for the final version of the publication, or visit the $\mathrm{DOI}$ to the publisher's website.

- The final author version and the galley proof are versions of the publication after peer review.

- The final published version features the final layout of the paper including the volume, issue and page numbers.

Link to publication

\section{General rights}

Copyright and moral rights for the publications made accessible in the public portal are retained by the authors and/or other copyright owners and it is a condition of accessing publications that users recognise and abide by the legal requirements associated with these rights.

- Users may download and print one copy of any publication from the public portal for the purpose of private study or research.

- You may not further distribute the material or use it for any profit-making activity or commercial gain

- You may freely distribute the URL identifying the publication in the public portal.

If the publication is distributed under the terms of Article 25fa of the Dutch Copyright Act, indicated by the "Taverne" license above, please follow below link for the End User Agreement:

www.tue.nl/taverne

Take down policy

If you believe that this document breaches copyright please contact us at:

openaccess@tue.nl

providing details and we will investigate your claim. 


\section{TECHNOLOGICAL UNIVERSITY EINDHOVEN}

Department of Mathematics

The Netherlands

Memorandum 1981-02

Januari 1981

Some theorems on Gabor Operators

(Dedicated to L.J.F. Broer on the occasion of his retirement)

J. de Graaf 


\section{Abstract}

The object of this study is the class of closable Gabor operators. That is the set of operators which map a Gabor function (or "note") into a multiple of a Gabor function.

By using the Bargman space (sometimes called Bargman representation) some general properies of these operators are derived.

It is shown that the set of Gabor operators whose adjoint is also a Gabor operator establishes a six dimensional complex manifold with a partial Lie-group structure and with an involution.

The corresponding Lie-algebra and the infinitesimal generators are calculated. Further it turns out that, at least locally, a Gabor operator with a Gabor adjoint results from an evolution process. The proofs of the theorems are a hybridization of Hilbext space techniques and classical complex analysis (Theorems of 0sgood, Montel, etc.). The proofs will be published elsewhere in a wider context.

\section{Introduction}

In physics, signal theory and music the so-called Gabor function

$$
g_{a, \omega, \gamma}(t)=\exp \left\{-{\left.\frac{(t-a)^{2}}{2 \gamma}+i \omega t\right\}}^{2}\right.
$$

with a:w, $\gamma \in \mathbb{R}$ and $\gamma>0$, plays an important rôle.

This function describes an impure tone of width $2 \sqrt{2 \gamma}$ at time a and angular frequency $\omega$. In music it could be the performance of a note of length $2 \sqrt{2 \gamma}$. Gabor functions are eigenfunctions of the eigenvalue problem

$$
\gamma \frac{d u}{d t}+t u=\lambda u
$$

Each $\lambda \in \mathbb{C}$ is an eigenvalue and $\lambda=a+i \gamma \omega$. For $\gamma=1$ our operator is just the annihilation operator and the corresponding Gabor functions are the socalled coherent states. 
I am interested in a description of all linear signal processors which turn each Gabor function into (a multiple of) a Gabor function. Mathematically this problem can be cast in the following form: Try to find or characterize all closable, densely defined, possibly unbounded operators in $I_{2}(\mathbb{R})$ which turn a Gabor function into a multiple of a Gabor function. operators having these properties will be called Gabor operators.

\section{Pxeliminaries}

I will use the Bargman space $V$. This is a Hilbert space of entire analytic functions. The inner product is

$$
(f, g)=\int \overline{f(z) g(z) d \mu(z) .}
$$

Here $d \mu(z)=\pi^{-1} \exp \left(-|z|^{2}\right) d x d y$. If not mentioned otherwise integrations are carried out over the whole of $\mathbb{a}$ considered as $\mathbb{R}^{2}$.

In $V$ one has the principal vectors $e_{w}(z)=\exp \bar{w} z$ with the property $\left(e_{W}, f\right)=f(w)$ for all $w \in \mathbb{C}$. Next, following Bargman, I introduce the mapping $A: \psi \mapsto f$ from $I_{2}(\mathbb{R})$ into $V$, defined by

$$
f(z)=(A \psi)(z)=\int_{-\infty}^{\infty} A(z, q) \psi(q) d q
$$

with $A(z, q)=\pi^{\frac{1}{2}} \exp \left\{-\frac{1}{2}\left(z^{2}+q^{2}\right)+\sqrt{2} z q\right\}$. This mapping $A$ is a bijective isometry. It maps the orthonormal Hermite basis of $I_{2}(\mathbb{R})$ into the orthonormal basis $\left\{(n !)^{-\frac{1}{2}} z^{n}\right\}$ of $V$. The expansion of an element $u$ in $V$ with respect to the last mentioned basis is easily obtained from the Taylor expansion of $u$.

Further, the mapping $A$ establishes a unitary isomorphism between the linear operators in $V$ and those in $L_{2}(\mathbb{R})$, namely $M=A^{-1} L A$. Here $L$ is an operator in $V$ and $M$ the corresponding operator in $L_{2}(\mathbb{R})$. The domains $D(L)$ and $D(M)$ are related by $D(M)=A^{-1} D(L)$. Some examples of operators in $L_{2}(\mathbb{R})$ together with their counterpart in $V$ are: The annihilation operator $M=2^{-\frac{1}{2}}(x+d / d x)$, $L=d / d z$. The creation operator $M=2^{-\frac{1}{2}}(x-d / d x), L=z$. The Fourier operator $M=F,(L u)(z)=u(i z)$. 
Any bounded operator $L$ in $V$ can be represented as an integral transform. For any $f \in V$ we have $(L f)(w)=\left(e_{w}, L f\right)=\left(I^{*} e_{w}, f\right)$, so that

$$
(L f)(w)=\int L(w, z) f(z) d \mu(z),
$$

where $L(w, z)=\overline{L^{*} e_{w}(z)}=\overline{\left(e_{z}, L^{*} e_{w}\right)}=\overline{\left(\operatorname{Le}_{z}, e_{w}\right)}$. Or $L(w, z)=\left(e_{w}, L e_{z}\right)=\left(L e_{z}\right)(w)$.

For the results of this section I refer to [B]. A short and very readible account of the Bargman space theory can be found in [J], pp. 215-218.

Finally calculation of the Bargman representation of a Gabor function yields

$$
\left(A g_{a, w, \gamma}\right)(z)=\varphi(a, w, \gamma) \exp \left\{\frac{1}{2}(\gamma-1)(\gamma+1)^{-1} z^{2}+2^{\frac{1}{2}} \gamma(\gamma+1)^{-1}\left(a \gamma^{-1}+1 \omega\right) z\right\}
$$

where the "phase factor" is

$$
\varphi(a, \omega, \gamma)=\pi^{-\frac{1}{4}}(2 \pi \gamma)^{\frac{1}{2}}(\gamma+1)^{-\frac{1}{2}} \exp \left\{-\frac{1}{2} \gamma^{-1} a^{2}+\frac{1}{4}\left(\gamma^{-1} a+i \omega\right){ }^{2}\right\} .
$$

This is an eigenfunction with eigenvalue $a+i \omega \gamma$ of the operator $2^{-\frac{1}{2}}\{(1-\gamma) z+(1+\gamma) d / d z\}$ defined in $V$. It is clear that the eigenfunction belongs to $V$ whenever $\left|(\gamma-1)(\gamma+1)^{-1}\right|<1$. That means $\operatorname{Re} \gamma>0$. Next we throw away the phase factors and define a Gabor function in $V$ to be the function

$$
e_{\sigma, w}(z)=\exp \left\{\frac{1}{2} \sigma z^{2}+\overline{w z}\right\}
$$

with $|\sigma|<1$. Obviously $e_{\sigma, w}$ is an eigenfunction with eigenvalue $\bar{w}$ of the operator $-\sigma z+d / d z$. In case $\sigma=0$ one retains the annihilation operator in the Bargman representation and $\left\{e_{0, w}\right\}, w \in \mathbb{C}$, are the coherent states. Now our mathematical problem is the following: Characterize the set of densely defined and closable operators in $V$ which map a Gabor function in a multiple of a Gabor function. From now on these operators will be called Gabor operators. 


\section{Some results on general Gabor operators}

As mentioned in the preceding section each element in $V$ can be written as a continuous superposition of the coherent states $e_{w}=e_{0, w}$

$$
u(w)=\int \overline{e^{\bar{w} z}} u(z) d \mu(z)
$$

Intuitively one suspects that the action of an operator $G$ is determined by $i t s$ action on the elements $e_{w}$. Heuristically

$$
(G u)(w)=\int\left(G_{z}\right)(w) u(z) d \mu(z)
$$

Necessary conditions for this expression to have a meaning is that $e_{z} \in D(G)$ for all $z \in \mathbb{C}$ and some sort of convergence of the integral.

\section{Definition 1:}

Let $f, g, h$ be mappings from $\mathbb{C}$ into itself. $f, g, h$ are not necessarily continuous or even measurable. Suppose $|h(z)|<1$ for all $z \in \mathbb{a}$. On the linear span $\left\langle e_{w}\right\rangle, i . e$. the set of finite linear combinations of the functions $e_{w^{\prime}}$ we define the Gabor operator $G_{f g h}$ by

$$
\left(G_{f g h} e_{w}\right)(z)=f(\bar{w}) \exp \left\{g(\bar{w}) z+\frac{1}{2} h(\bar{w}) z^{2}\right\}
$$

and linear algebraic extension.

Algebraically speaking the functions $e_{w^{\prime}} w \in \mathbb{C}$, are independent but topologically they are not. So it is not surprising that imposing a topological condition on $G_{f g h}$ implies some smoothness of the functions $f, g, h$.

\section{Theorem 2:}

A necessary condition for the operator $G_{f g h}$ to be closable is that $f, g$ and $h$ are analytic functions on an open and dense subset of $\mathbb{c}$.

A question that immediately comes up is: Does it follow from the closability of $G_{f g h}$ that $f, g$ and $h$ are entire functions? I don't know whether 
this follows from the closability condition alone. However, with a gentle additional condition it follows that $f, g$ and $h$ are entire analytic functions.

\section{Theorem 3:}

Suppose that $G_{f g h}$ is closable and suppose that $f$ and $g$ map bounded sets into bounded sets. Then $h$ must be constant and $f$ and $g$ must be entire functions.

The condition on $f$ and $g$ in this theorem is satisfied if $f$ and $g$ are supposed to be continuous or if $G_{f g h}$ is supposed to be a bounded operator in $V$. The operator $G_{f g h}$ is closable iff the domain $D\left(G^{*}{ }_{f g h}\right)$ of its adjoint $G_{f g h}^{\star}$ is dense. If we are a little specific on $D\left(G_{f g h}^{\star}\right)$ then it also follows that $f, g$ and $h$ are entire. This is done in the next theorem.

\section{Theorem 4:}

A) $\left\{z^{n}\right\}_{n=0}^{\infty} \subset D\left(G_{f g h}^{*}\right)$, i.e. the domain of the adjoint contains all polynomials, if and only if $f$ and $g$ are entire, $h$ is constant and $\mathrm{fg}^{\mathrm{n}} \in V$ for $\mathrm{n}=0,1,2, \ldots$.

B) $\left\{e_{\alpha}\right\}_{\alpha \in \mathbb{C}} \subset D\left(G_{f g h}^{*}\right)$, i.e. the domain of the adjoint contains all coherent states, if and only if $\overline{f(\bar{w})} \exp \left[\overline{g(\bar{w})} z+\frac{1}{2} \overline{h(\bar{w})} z^{2}\right\}$ for each fixed $z \in \mathbb{C}$, as a function of $w$, belongs to $V$.

C) The conditions on $f, g, h$ as mentioned in $A$ ) and $B$ ) are sufficient for $\mathrm{G}_{\text {fgh }}$ to be closable.

In the sequel I consider closable Gabor operators with $f$ and $g$ entire functions and $h$ a complex constant, $|h|<1$. The next theorem contains some information on the domain $D\left(\bar{G}_{f g h}\right)$ of the closure $\bar{G}_{f g h}$ of $G_{f g h}$ and also on $D\left(G_{f g h}^{*}\right)$.

\section{Theorem 5:}

A) Let $\Omega \subset \mathbb{C}$ be a compact subset. Let $\psi \in L_{1}(\Omega)$. Then $\int_{\Omega} \psi(w) e^{\bar{w} Z} d \mu(w)$, as a function of $z$, belongs to $D\left(\bar{G}_{f g h}\right)$. 
B) Let $u \in V$. Suppose

$$
\lim _{R \rightarrow \infty} \int_{|w|<R} f(\bar{w}) \exp \left\{g(\bar{w}) z+\frac{1}{2} h z^{2}\right\} u(w) d \mu(w)
$$

exists in $V$-sense. Then $u \in D\left(\bar{G}_{f g h}\right)$ and the limit is equal to $\bar{G}_{f g h} u$. C) Let $v \in D\left(G_{f g h}^{*}\right)$. Then

$$
\left(G_{f g h}^{*} v\right)(\alpha)=\int \overline{f(\bar{s})} \exp \left\{\overline{g(\bar{\alpha}) \bar{w}}+\frac{1}{2} \bar{h}^{2}\right\} v(w) d \mu(w) .
$$

Any bounded operator in $V$ can be represented by a kernel, see [B]. In the next theorem a class of unbounded closes operators is introduced which can be represented by a kernel. The result is then applied to Gabor operators.

\section{Theorem 6:}

Let $A$ be a densely defined operator in $V$ and suppose $D(A)>\left\{e_{\alpha}\right\}$, $D\left(A^{*}\right) \supset\left\{e_{\alpha}\right\}$. Then there exists an entire function $a(u, v)$ on $e^{2^{\alpha}}$ such that for each $\in \mathcal{D}(\mathrm{A})$

$$
(A \varphi)(z)=\int a(z, \bar{w}) \varphi(w) d \mu(w)
$$

For each $u \in \mathbb{C} a(u,:) \in V$ and for each $v \in \mathbb{a} a(\cdot, v) \in V$.

\section{Theorem 7:}

Suppose $G$ is an injective Gabor operator such that $D\left(G^{*}\right)=\left\{e_{\alpha}\right\}$. Then $G^{*}$ is also a Gabor operator and the kernel of $G$ is given by

$$
a(z, \bar{w})=\exp \left\{A_{0}+\frac{1}{2} A_{1} w^{-2}+A_{2} \bar{w}+A_{3} \bar{w} z+A_{4} z+\frac{1}{2} A_{5} z^{2}\right\}
$$

where $A_{i}, 0 \leq i \leq 5$, are complex constants, $\left|A_{1}\right|<1,\left|A_{5}\right|<1$.

\section{Remark 8:}

If both $G$ and $G^{*}$ are Gabor operators, the injectiveness of $G$ are $G^{*}$ follows. 
4. On the composition of Gabor operators whose adjoint is also a Gabor operator

Let $D \subset \mathbb{C}$ denote the open unit disc. Consider the manifold

$\Gamma=\mathbb{C} / 2 \pi i \times D \times \mathbb{C} \times \mathbb{C} \backslash\{0\} \times \mathbb{C} \times D$. Denote $\underline{A}=\left(A_{0}, A_{1}, \ldots, A_{5}\right) \in \Gamma$.

with each $\underline{A} \in \Gamma$ we associate a Gabor operator $G_{\underline{A}}$ by

$$
\left(G_{\underline{A}} u\right)(z)=\int g(\underline{A}, z, \bar{w}) u(w) d u(w)
$$

with

$$
g(\underline{A}, z, \bar{w})=\exp \left\{A_{0}+\frac{1}{2} A_{1} \bar{w}^{-2}+A_{2} \bar{w}+A_{3} \bar{w} z+A_{4} z+\frac{1}{2} A_{5} z^{2}\right\} .
$$

From the preceding section it follows that $G_{\underline{A}}^{*}=G_{\underline{A}}$ with $\underline{\underline{A}}=\left(\bar{A}_{0}, \bar{A}_{5}, \overline{\mathrm{A}}_{4}, \overline{\mathrm{A}}_{3}, \overline{\mathrm{A}}_{2}, \overline{\mathrm{A}}_{1}\right)$. All functions $e_{\alpha}, \alpha \in \mathbb{C}$, are in the domain of each $G_{A}$ but in general functions of type exp $\left\{\bar{\alpha} z+\frac{1}{2} \bar{\beta} z^{2}\right\}$ are only in $D\left(G_{A}\right)$ if $|\bar{\beta}|$ is small enough. Since application of $G_{A}$ to some $e_{\alpha}$ may lead to the exponential of a $2^{\text {nd }}$ degree polynomial, the composition $G_{A} \circ G_{B}$ is not always an operator with each $e_{\alpha}$ in its domain. If for given $\bar{B}$ the constants $A_{1}, \ldots, A_{5}$ are chosen sufficiently close to $0,0,1,0,0$, composition is possible such that each $e_{\alpha}$ is in the domain of $G_{\underline{A}} \cdot G_{B}$.

\section{Definition 9:}

$r$ is endowed with a partial Lie-group structure and with an involution in the following way.

The composition law is

$$
\begin{aligned}
& \underline{A} * \underline{B}=\left(A_{0}, A_{1}, \ldots, A_{5}\right) *\left(B_{0}, B_{1}, \ldots, B_{5}\right)= \\
& \left(B_{0}+A_{0}+\frac{1}{2}\left(A_{1} B_{4}^{2}+B_{5} A_{2}^{2}+2 B_{4} A_{2}\right)\left(1-A_{1} B_{5}\right)^{-1}-\frac{1}{2} \log \left\{1-A_{1} B_{5}\right\}\right. \\
& B_{1}+A_{1} B_{3}^{2}\left(1-A_{1} B_{5}\right)^{-1}, B_{2}+A_{1} B_{3} B_{4}\left(1-A_{1} B_{5}\right)^{-1}+ \\
& B_{3} A_{2}\left(1-A_{1} B_{5}\right)^{-1}, B_{3} A_{3}\left(1-A_{1} B_{5}\right)^{-1}, \\
& A_{4}+B_{5} A_{2} A_{3}\left(1-A_{1} B_{5}\right)^{-1}+A_{3} B_{4}\left(1-A_{1} B_{5}\right)^{-1}, \\
& \left.A_{5}+B_{5} A_{3}^{2}\left(1-A_{1} B_{5}\right)^{-1}\right) .
\end{aligned}
$$

The involution is

$$
\underline{\tilde{A}}=\left(A_{0}, A_{1}, \ldots, A_{5}\right)^{\sim}=\left(\bar{A}_{0}, \bar{A}_{5}, \bar{A}_{4}, \bar{A}_{3}, \bar{A}_{2}, \bar{A}_{1}\right) .
$$




\section{Remarks 10:}

1) The expression ( $(t)$ makes sense for each $\underline{A}, \underline{B} \in \Gamma$. The result of the composition however is not necessarily an element of $\Gamma$. For given $\underline{A}$ composition with an element $\underline{B}$ sufficiently near to $(0,0,0,1,0,0)$ leads to an element in $F$.

2) $(\underline{A} * \underline{B})^{\sim}=\underline{\tilde{B}} * \underline{\tilde{A}}$.

3) $(0,0,0,1,0,0)$ acts as a unit element.

4). The left inverse of $\underline{B}$ is given by

$$
\begin{aligned}
& \left(A_{0} B_{1}\left(B_{1} B_{5}-B_{3}^{2}\right)^{-1},\left(B_{3} B_{2}-B_{1} B_{4}\right)\left(B_{1} B_{5}-B_{3}^{2}\right)^{-1},\right. \\
& -B_{3}\left(B_{1} B_{5}-B_{3}^{2}\right)^{-1},\left(B_{3} B_{4}-B_{2} B_{5}\right)\left(B_{1} B_{5}-B_{3}^{2}\right)^{-1}, \\
& \left.B_{5}\left(B_{1} B_{5}-B_{3}^{2}\right)^{-1}\right) .
\end{aligned}
$$

$A_{0}$ can be calculated by putting the first component in $(t)$ equal to zero and substitution of the known variables. Obviously the inverse exists. On a sufficiently small open neighbourhood of the unit element the inverse is again an element of $\Gamma$.

5) With the composition rule we introduced above the elements $\underline{A} \in \Gamma$ for which $A_{1}=A_{5}=0$ establish a Lie-group.

\section{Theorem 11:}

Suppose that the product operator $G_{A_{A}} \circ G_{B}$ contains all coherent states $e_{\alpha^{\prime}} \alpha \in \mathbb{C}$, in its domain, then $G_{\underline{A}} \circ{ }^{\circ} G_{B}=G_{A} * B_{B}$.

The variable $\mathrm{A}_{0}$ only plays a subordinate role. It has to do with phase factors. Therefore I introduce the manifold

$$
\Sigma=D \times \mathbb{C} \times \mathbb{C} \backslash\{0\} \times \mathbb{C} \times D
$$

The notation of the variables is $\underline{x}=\left(x_{1}, \ldots, x_{5}\right)$.

With the composition $(t)$ where the first component is left out, $\Sigma$ is again a partial Lie-group with involution. The tangent bundle $\mathrm{T} \Sigma$ is trivializable. A right invariant vectorfield $\underline{v}(\underline{x})$ is a vectorfield with the property

$$
\frac{\partial}{\partial \underline{x}}(\underline{x} * \underline{y}) \cdot \underline{v}(\underline{y})=\underline{v}(\underline{x} * \underline{y})
$$


For each $y$ there is an open neighbourhood $\Omega_{y}$ of unity such that this expression makes sense for each $\underline{x} \in \Omega_{\underline{y}}$.

Denote the tangent space at the unit element $\underline{e}^{b y} \mathrm{~T}_{\mathbf{e}^{\Gamma}}$.

Let $\underline{\alpha}=\left(\alpha_{1}, \ldots, \alpha_{5}\right) \in \mathrm{T}_{\mathrm{e}^{\Sigma}}$. Then a right invariant vectorfield is given by

$$
\underline{v}(\underline{x})=\underline{\theta}(\underline{x}) \cdot \underline{\alpha}=\left[\begin{array}{ccccc}
x_{3}^{2} & 0 & 0 & 0 & 0 \\
x_{4} x_{3} & x_{3} & 0 & 0 & 0 \\
x_{5} x_{3} & 0 & x_{3} & 0 & 0 \\
x_{5} x_{4} & x_{5} & x_{3} & 1 & 0 \\
x_{5}^{2} & 0 & 2 x_{5} & 0 & 1
\end{array}\right]\left[\begin{array}{l}
\alpha_{1} \\
\alpha_{2} \\
\alpha_{3} \\
\alpha_{4} \\
\alpha_{5}
\end{array}\right] .
$$

Obviously $\underline{v}(0,0,1,0,0)=\left(\alpha_{1}, \ldots, \alpha_{5}\right)$

One-parameter partial sub-Lie-groups of $\Sigma$ are obtained by solving the differential equation

$$
\frac{\mathrm{d} \underline{\underline{x}}}{\mathrm{dt}}=\underline{\underline{\theta}} \cdot \underline{\alpha} \quad \text { with } \underline{x}(0)=\underline{e} .
$$

The tangent space $\mathrm{T}^{\Sigma}$ at the unit element is made into a Lie-algebra in the usual way the $\frac{e}{i d}$ of the local formula for the lie-product

$$
[\underline{u}(\underline{x}) \cdot \underline{v}(\underline{x})]=D \underline{u}(\underline{x}) \cdot \underline{v}(\underline{x})-D \underline{v}(\underline{x}) \cdot \underline{u}(\underline{x}) .
$$

Evaluation of this lie-product leads to the expression

$$
\begin{aligned}
{[\underline{\alpha}, \underline{\beta}]=} & \operatorname{column}\left(2\left(\alpha_{1} \beta_{3}-\beta_{1} \alpha_{3}\right),\left(\alpha_{2} \beta_{3}-\beta_{2} \alpha_{3}\right)+\left(\alpha_{1} \beta_{4}-\beta_{1} \alpha_{4}\right),\right. \\
& \left.\alpha_{1} \beta_{5}-\beta_{1} \alpha_{5},\left(\alpha_{3} \beta_{4}-\beta_{3} \alpha_{4}\right)+\left(\alpha_{2} \beta_{5}-\beta_{2} \alpha_{5}\right), 2\left(\alpha_{3} \beta_{5}-\beta_{3} \alpha_{5}\right)\right) .
\end{aligned}
$$


Theorem 12:

Consider the set $G$ of Gabor operators whose adjoint is also a Gabor operator. Let $G$ be provided with the operations of composition (whenever defined on the whole of span $\left.\left\langle e_{\alpha}\right\rangle\right\rangle$ and taking the adjoint. Then $G$ is a bijective representation of the partial Lie-group with involution $\Gamma$. By this I mean:

(i) The mapping $\Gamma \ni \underline{A} \vdash G_{A}$ is bijective.

(ii) $G_{\underline{A}} \circ G_{B}=G_{\underline{A}} * \underline{B}$. The left-hand side is defined iff the right-hand side is defined.

(iii) $\mathrm{G}_{\underline{\mathrm{A}}}^{\star}=\mathrm{G}_{\tilde{\mathrm{A}}}^{\sim}$.

(iv) If $\mathrm{P}(t \overline{)},-\varepsilon<t<\varepsilon, \varepsilon>0, \mathrm{P}(0)=\mathrm{I}$, is a one-parametex partial subgroup of $G$, then $P(t)$ has an infinitesimal generator of the form

$$
\frac{1}{2} \alpha_{1} \frac{d^{2}}{d z^{2}}+\alpha_{2} \frac{d}{d z}+\alpha_{3} z \frac{d}{d z}+\alpha_{4} z+\frac{1}{2} \alpha_{5} z^{2}
$$

with $\alpha_{1}, \ldots, \alpha_{5} \in \mathbb{C}$.

(v) The set of differential operators of the form mentioned in (iv) can be made into a Iie-algebra in the usual way by defining the tie-product equal to the commutator. This Lie-algebra is isomorphic to the Lie-algebra $T_{\underline{e}}{ }^{\Sigma}$.

(vi) There is a neighbourhood $\Omega$ of $e$ in $\Sigma$ such that each element in $\Omega$ is an element of a one-parameter partial subgroup of $\Sigma$. In the set of Gabor operators corresponding to $\Omega$ each Gabor operator is the result of an evolution process.

\section{Final remarks}

a) I don't know whether (vi) of the last theorem is globaily true. That is whether each Gabor operator that has a Gabor adjoint results from one evolution process.

b) The translation from $V$ back to $\mathrm{L}_{2}$ ( $\mathbb{R}$ ) canibe made via (iv) of the last theorem. The set of infinitesimal generators has exactly the same form there. This can be seen by using the translation rules for creation 
and annihilation operators as mentioned in the Preliminaries.

c) The class of operators mentioned by De Bruijn $[\mathrm{Br}]$, section 27.3, consists of Gabor operators whose adjoint is also Gabor operator. It is a proper subset of the class of operators I discussed in the last section.

\section{References}

[J] Jauch, J.M., Foundations of Quantum Mechanics Addison-Wesley. Reading, Massachusetts 1968.

[B] Bargmann, V., On a Hilbert Space of analytic functions and an associated integral transform. Comm. Pure Appl. Math. 14 (1961), 187-214.

[BY] Bruijn, N.G. De., A theory of generalized functions with applications to Wigner distribution and Weyl correspondence. Nieuw Archief voor Wiskunde 21 (1973) 205-280. 